\title{
The Pathophysiology of Primary Hip Osteoarthritis may Originate from Bone Alterations
}

\author{
Mikio Kamimura ${ }^{1}$, Yukio Nakamura ${ }^{2,3,{ }^{*}}$, Shota Ikegami $^{2}$, Keijiro Mukaiyama $^{2}$, \\ Shigeharu Uchiyama ${ }^{2}$ and Hiroyuki Kato ${ }^{2}$ \\ ${ }^{1}$ Center of Osteoporosis and Spinal Disorders: Kamimura Orthopaedic Clinic, Matsumoto, 399-0021, Japan \\ ${ }^{2}$ Department of Orthopaedic Surgery, Shinshu University School of Medicine, Asahi3-1-1, Matsumoto, 390-8621, Japan \\ ${ }^{3}$ Department of Orthopaedic Surgery, Showa Inan General Hospital, Komagane, 399-4117, Japan
}

\begin{abstract}
Objectives: The aim of this study was to investigate whether bone alterations detected by hip magnetic resonance imaging (MRI) were associated with subsequent primary hip OA.

Methods: We enrolled 7 patients with hip joint pain from their first visit, at which hip joints were classified as grade 0 or I on the Kellgren-Lawrence grading scale. Plain radiographs and magnetic resonance imaging (MRI) were performed on all cases, and pain was assessed with the Denis pain scale. Average age, height, weight, body mass index, bone mineral density (L1-4), central edge angle, Sharp's angle, and acetabular hip index were calculated.

Results: Within two months of the onset of pain, 4 of the 7 cases showed broad bone signal changes, while 3 cases showed local signal changes in the proximal femur on hip MRI. Three to 6 months after the onset of pain, in all patients whose pain was much improved, plain radiographs showed progression to further-stage OA.

Conclusion: Our findings suggest that bone abnormalities in the proximal femur might be involved in the pathogenesis of primary hip OA.
\end{abstract}

Keywords: Pathophysiology, bone, hip osteoarthritis, MRI, joint pain.

\section{INTRODUCTION}

Osteoarthritis $(\mathrm{OA})$ is the most common musculoskeletal disorder in elderly people, and it is generally accepted that continuous mechanical stress causes articular cartilage degradation. However, several decades ago, Radin and Rose reported that $\mathrm{OA}$ could be initiated and could progress from subchondral bone [1]. Furthermore, Burr and Gallant proposed that subchondral bone remodeling might be the main pathophysiology of OA [2]. Recently, Guermazi and colleagues reviewed that $\mathrm{OA}$ is considered a whole-joint disease process [3]. $\mathrm{Xu}$ and colleagues reviewed that bone marrow signal alterations are a common magnetic resonance imaging (MRI) feature of OA. However, all of the data were based on patients with advanced OA [4]. Guermazi and colleagues performed a population-based observational study and found that abnormalities were detected by knee MRI in $89 \%$ of people $>50$ years of age who had no plain radiographic evidence of OA. The limitation of their study was that they did not undertake further imaging evaluation [5]. We have shown that MRI examination reveals that bone signal changes are detectable in patients with hip OA at all

*Address correspondence to this author at the Department of Orthopaedic Surgery, Shinshu University School of Medicine, Asahi3-1-1, Matsumoto, 390-8621, Japan; Tel: +81-263-37-2659; Fax: +81-263-35-8844;

E-mail: yxn14@aol.jp stages with joint pain, while broad bone signal changes are not detectable if the patients do not have hip joint pain [6]. As far as we know, no report has examined the relationship among joint pain, signal changes detected by MRI, and radiographic evaluation of $\mathrm{OA}$ progression in patients without OA or with a slight degree of OA.

In this study, we aimed to examine whether bone abnormalities may contribute to the pathophysiology of primary hip OA. Toward this goal, we enrolled 7 patients with acute hip joint pain, without any traumatic events or advanced hip OA. We then performed hip MRI and plain hip radiographs on all of the patients, who were thereafter observed by plain radiographs. Based on these findings, we investigated the relationship between bone alterations and OA progression in the hip joints.

\section{PATIENTS AND METHODS}

In this study, none of the 7 enrolled patients had recognized any prior hip joint pain, and their plain radiographs were classified as grade 0 or I on the KellgrenLawrence (KL) grading scale [7]. In all patients, hip joint pain occurred without any traumatic episode. Six of the 7 patients did not show any evidence of acetabular dysplasia; one patient showed a mild acetabular dysplasia (case 2), and another patient had a Perthes-like joint deformity (case 6). Except for the patient with the Perthes-like joint deformity, 
Table 1. Parameters Determined in the 8 Patients with Primary Hip OA

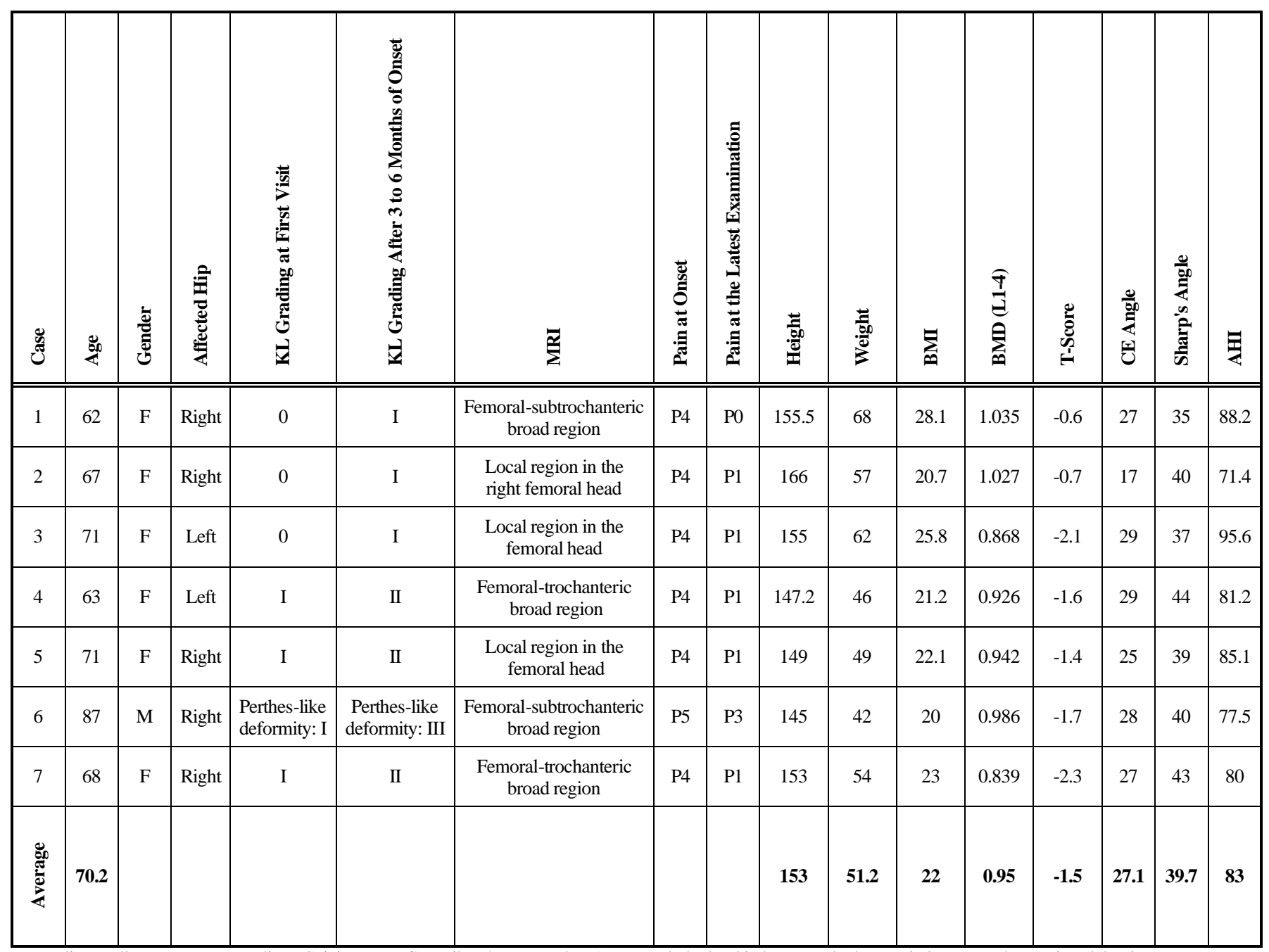

KL grading: Kellgren-Lawrence grading; JS: joint space; CE: Wiberg's center-edge; AHI: acetabular head index; BMI: body mass index; BMD: bone mineral density.

the average of Wiberg's center-edge (CE) angle was $27.1^{\circ}$, Sharp's angle was $39.7^{\circ}$, and acetabular head index (AHI) was 82.7 (Table 1).

The patient with the Perthes-like joint deformity had already undergone total hip replacement surgery on the left hip (case 6) (Table 1).

All patients were treated with non-steroidal antiinflammatory drugs and/or tramadol. A plain radiograph was taken at the first visit and at 3 to 6 months after the onset of pain, and a hip MRI examination was performed within two months of the onset of pain.

When low intensity by T1-weighted image (T1W) and high intensity by short-tau inversion recovery T2-weighted image (T2-STIR) were detected in the hip joint, we noted that signal changes were observed. Bone mineral density (BMD) was measured using a Dual-energy X-ray Absorption (DXA) fan-beam bone densitometer (Lunar Prodigy; GE Healthcare, Waukesha, WI, USA) at the L1-4 levels of the posteroanterior spine. Pain was assessed with the Denis pain scale [8], on which $\mathrm{P} 1=$ no pain; $\mathrm{P} 2=$ occasional minimum pain with no need for medication; P3=moderate pain with occasional medication but no interruption of work or significant changes in activities of daily living (ADL);
$\mathrm{P} 4=$ moderate to severe pain with frequent medication and occasional absence from work or significant change in ADL; and $\mathrm{P} 5=$ constant or severe incapacitating pain requiring chronic medication. The current study was approved by the institutional ethical committee, and informed consent was obtained from all of the patients.

\section{RESULTS}

Six of 7 patients were female, and one was male. The average age (years), height $(\mathrm{cm})$, body weight $(\mathrm{kg})$, and body mass index (BMI) $\left(\mathrm{kg} / \mathrm{m}^{2}\right)$ were $70.2,153.0,51.2$, and 22.0, respectively. All patients were generally in good physical condition and did not have a history of any serious complications. Their joint pain occurred mainly while walking, without any traumatic events. All 7 cases were without advanced radiographic $\mathrm{OA}$ features at their first visit. Two patients who were diagnosed with mild osteoporosis had already received osteoporosis treatment before the hip MRI was performed.

All 7 patients first visited our institutions with a complaint of acute hip joint pain. In all 7 cases, the hip pain improved in a few months. 
Within two months of the onset of pain, hip MRI was performed and revealed that all 7 cases showed low intensity by $\mathrm{T} 1 \mathrm{~W}$ and high intensity by T2-STIR in the proximal femur. In 4 cases, broad signal changes, which were not only in the burdened areas, but also in the broad regions from the femoral head to the trochanters, were detected by hip MRI. In the 3 remaining cases, local signal changes, which were in part of the femoral head, were observed on hip MRI. Three to 6 months after the onset of pain, plain radiographs of all patients whose pain had been much improved, showed progression of OA according to the KL grading scale (Table 1).

There are 2 representative cases as follows:

Patient 1 is a case without radiographic OA patient but with a joint pain. After broad bone signal changes by hip MRI, radiograhically OA proceeded.

Patinet 2 is a case without radiographic OA patient but with a joint pain. After local bone signal changes by hip MRI, radiograhically OA proceeded.

\section{CASES}

\section{Patient 1}

A 62-year-old woman, $155.5 \mathrm{~cm}$ tall and weighing 68.0 $\mathrm{kg}$, with no past history visited our hospital. At her first visit, she complained of slight locking symptoms with accompanying right hip pain after a long walk during a trip and bilateral knee pain while walking and reclining in a lateral recumbent position. Plain radiographs of the bilateral knee joints showed advanced-stage OA, while the right hip joint did not show OA features (KL grading 0, Fig. 1). Physical examination revealed restricted abduction range of motion (ROM) in her right hip. A month after the onset of pain, hip MRI showed broad and heterogeneous low intensity by T1W and high intensity by T2-STIR (Fig. 2a, b). Two months after onset, her joint pain disappeared; however, a slight joint space narrowing in the femoral head was found on plain radiographs (KL grading I, Fig. 3). Interestingly, her left hip joint, which was without pain, had already shown slight joint space narrowing, but no signal change on MRI (Figs. 1-3).

\section{Patient 2}

A 67-year-old woman, $166 \mathrm{~cm}$ tall and weighing $57 \mathrm{~kg}$, visited our hospital very recently. Her pain in the right hip joint started during gait without any episode. Plain radiographs showed a KL grading of 0 in the right hip (KL grading 0, Fig. 4). ROM in the right hip joint was full. A week after the onset of pain, hip MRI revealed femorallateral local bone alterations in the right hip joint (Fig. 5a, b). More joint space narrowing was seen in the right hip on a plain radiograph (KL grading I, Fig. 6). Her right hip pain is currently much reduced.
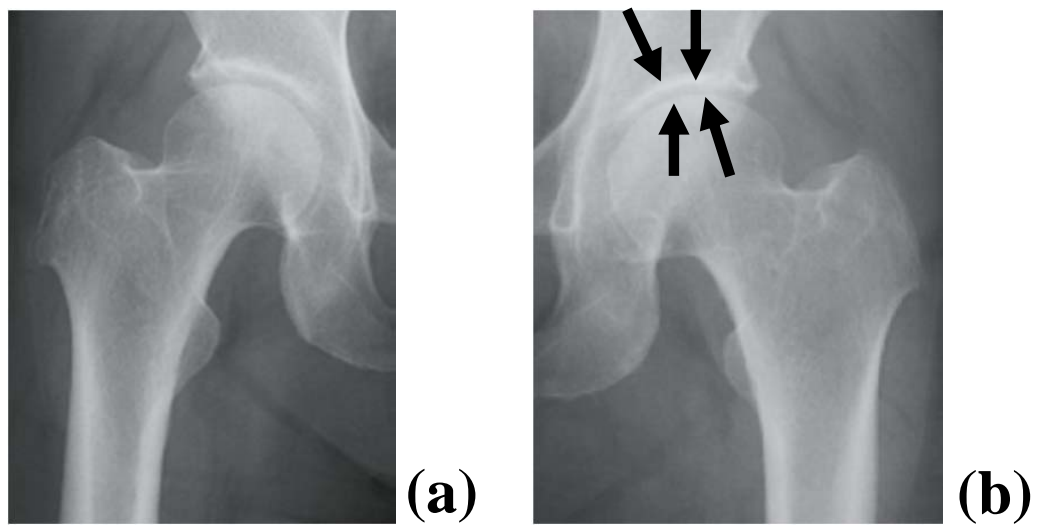

Fig. (1). At her first visit, the patient's KL grading was 0 in the right hip joint (a) and I in the left hip joint (b). No OA features were seen in the right hip (a), and a slight joint space narrowing was seen in the left hip joint (b, arrows) on a plain radiograph. Note that the patient complained of right hip pain.
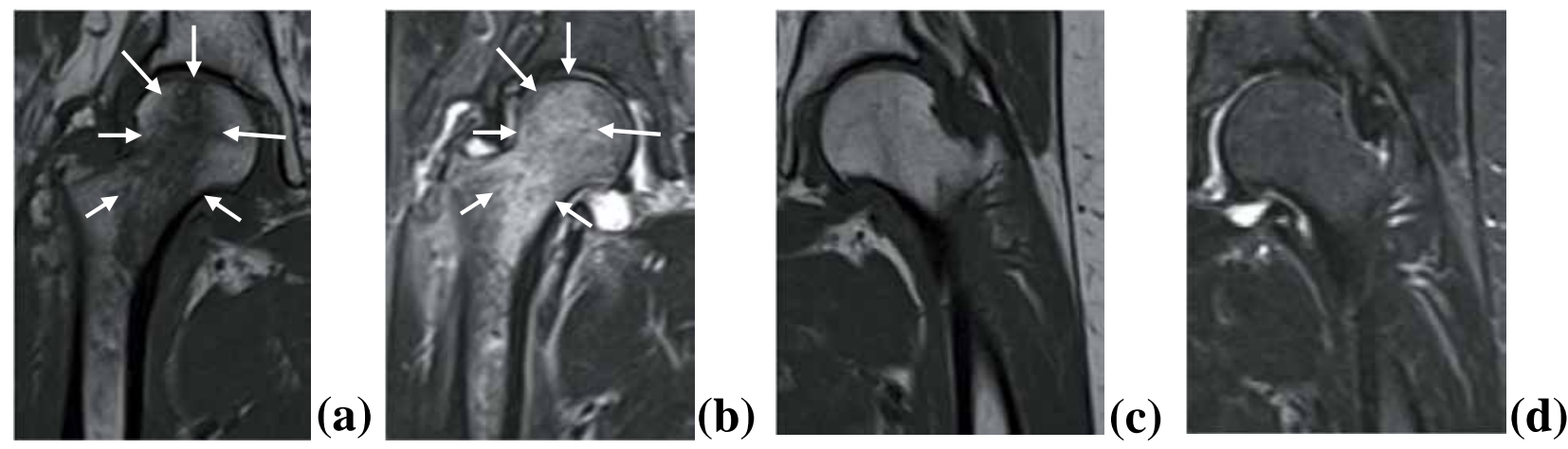

Fig. (2). A month after the onset of pain, MRI showed broad and heterogeneous signal changes. Low intensity by T1W (a, arrows) and high intensity by T2-STIR (b, arrows) were observed in the right hip, although no signal change was observed in the left hip joint. 

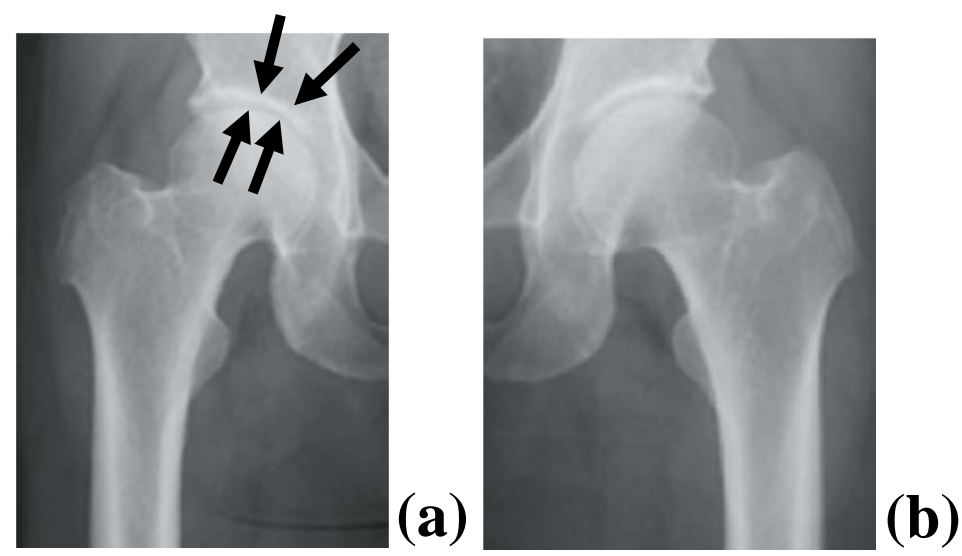

Fig. (3). The KL grading was I in the right hip with OA (a) and I in the left hip with OA (b). Two months after the onset of pain, a slight joint space narrowing (arrow) in the right hip joint was seen on a plain radiograph, although no radiographic change was seen in the left hip joint.

\section{DISCUSSION}

We showed that bone alterations confirmed by MRI in patients with joint pain were associated with subsequent progression to further-stage OA. The signal changes detected by MRI were distributed extensively on the burdened area of the femoral head in all patients. Of note, these bone alterations were difficult to detect on plain radiographs obtained during the first examination. Our findings suggest that one of the causes of hip OA may be pathological bone changes, which can be revealed by MRI. To the best of our knowledge, this is the first report to investigate the relationship among joint pain, MRI-revealing bone alterations, and hip OA progression confirmed by plain radiographs.

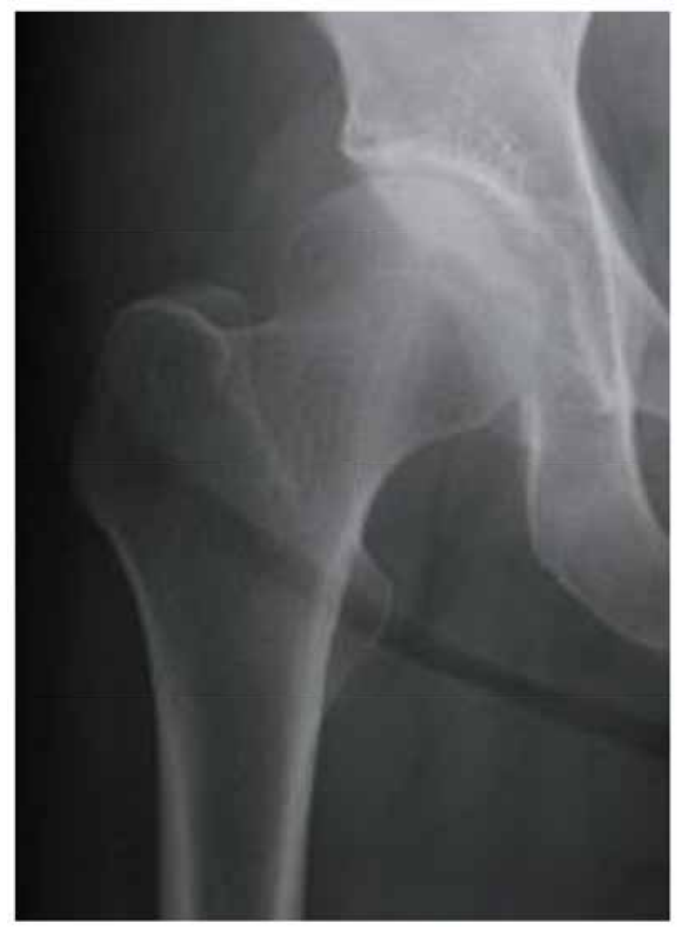

Fig. (4). At her first visit, the patient's KL grading was 0 in the right hip. No obvious OA was seen in the right hip on plain radiographs.
In our study, MRI analysis showed low intensity on T1W and high intensity on T2-STIR in all 7 cases associated with pain. Usually, low intensity on $\mathrm{T} 1 \mathrm{~W}$ and high intensity on T2-weighted images imply bone marrow edema or hemorrhage, and these findings are not specific to bone fractures. Signal changes on MRI in joints in cases with insufficiency fractures or bone microfractures have been reported [9-11]. Guermazi and colleagues recently reviewed that these signal changes of MRI in joints frequently suggest microfractures [3]. Previously, we demonstrated that a vertebral body with these MRI findings and severe back pain evolved into late collapse and/or sclerosis, while back pain was decreased, and we concluded that a low T1W showing a signal change for the vertebral body with back pain indicated the presence of a fracture [9]. In this study, 1) the broad signal changes were detected with joint pain, and 2) the joint pain improved in all cases. Similar to our previous report, it is conceivable that the signal changes detected by MRI in the femoral head with hip joint pain might be insufficiency fractures.

It has been reported that subchondral bone is important as a cause of OA $[1,12,13]$. Subchondral bone usually refers to both subchondral cancellous bone and the cortical plate, without making a proper distinction for their differences [13]. $\mathrm{Xu}$ and colleagues recently reported that bone marrow alterations detected by MRI are a common imaging feature of $\mathrm{OA}$ and that the alterations are associated with the progression of articular cartilage degeneration. However, all of the available data were derived from patients with advanced OA [4]. Burr and Gallant reviewed that the main pathophysiology of $\mathrm{OA}$ is bone remodeling in subchondral bone [2]. The authors have reported that subchondral cancellous bone microfractures cannot be the pathophysiological mechanism of OA progression, since cancellous bone is distant from articular cartilage. On the other hand, they reported that OA progression could be due to imbalanced bone remodeling, which leads to increased bone formation; however, the underlying mechanism is not known [13,14].

In this study, we revealed that, in cases without OA or with early hip OA, hip pain occurred without traumatic events, and bone alterations were detected by hip MRI. Hip 

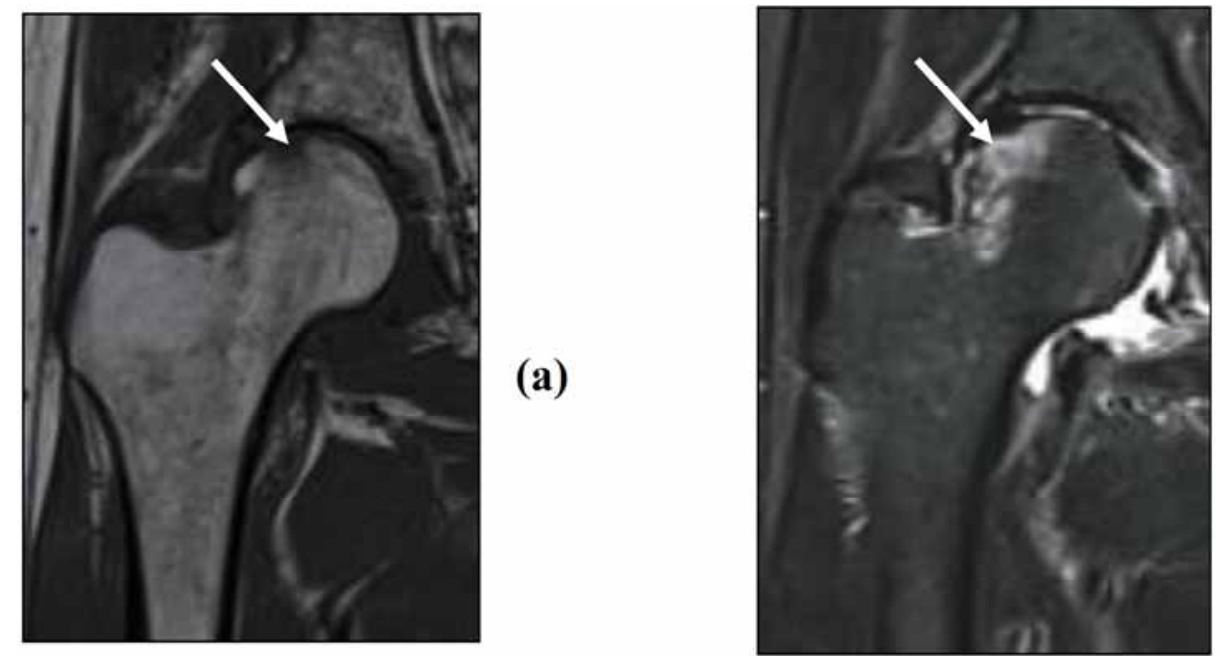

(b)

Fig. (5). MRI showed local signal changes in the right femoral-lateral head. Low intensity by T1W (a, arrow) and high intensity by T2- STIR (b, arrow) depicted bone alterations in the femoral-lateral head.

joint pain was thereafter improved in all cases. On the other hand, hip joint OA progressed to further-stage OA in all of the patients. Currently, the mechanism of this OA progression has not been identified. Our previous study showed that, in the cases with advanced OA, no signal change was likely when patients had no joint pain, while broad signal changes were likely when patients had joint pain [6]. These results indicate that bone alterations detected by MRI are associated with joint pain and OA progression, while OA progression and joint pain are not necessarily related to each other.

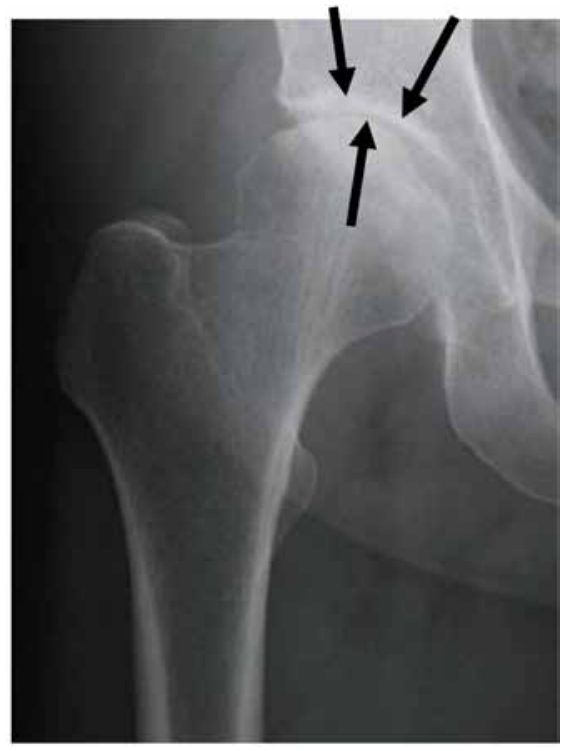

Fig. (6). The KL grading was I in the right hip. Even though the right hip joint pain disappeared, a slight joint space narrowing was seen in the right hip on a plain radiograph (arrows).

Given the findings of our two studies (this study and 6), there might be the following classifications for $\mathrm{OA}$ progression via bone alterations (Fig. 7). First, healthy joints progress to the MRI-based signal change stage for some reason: cases having joint pain without or with early radiographic OA changes, but bone signal changes detected by MRI accompanied with possible microfractures. We propose that this is the MRI-based signal change stage. Second, cases with joint pain that proceed to OA due mainly to bone involvement. In cases with few bone alterations, cartilage degeneration might be stagnant; consequently, pain could be improved or stabilized, even though patients have joint space narrowing on plain radiographs without bone signal changes by MRI. We propose that this is the remission-staged OA. In cases with significant bone alterations, cartilage and bone degradation continue with joint pain. We propose that this is the end-staged OA. Since the number of cases were relatively small in this study as well as our previous study [6], it is currently speculative the mechanism of OA progression (Fig. 7).

In case 1 and 2, the joint with pain had no OA features on plain radiographs and broad signal changes on MRI. This is thought to be the MRI-based signal change stage. This case thereafter was thought to become early-staged OA, since more joint space narrowing was observed on plain radiographs, and the pain disappeared. Interestingly, in the case 1 , the other joint without pain already had slight joint space narrowing and no signal change by MRI (Figs. 1, 2). Both of these joints are also thought to be early-staged OA.

It is generally well known that joint pain is not necessarily related to radiographic evaluation of OA based on KL grading scale. In the ROAD study, Muraki et al. first reported about KL grading scale in Japanese knee OA patients. The authors reported the existence of a cause of joint pain that is independent of OA in women, while the pain in men may be more dependent on joint space narrowing by $\mathrm{OA}$ according to KL grading [7]. These results may suggest that joint space narrowing might not be the hallmark for joint pain in OA patients. In our previous study, the bone signal change was not seen in the cases with advanced OA without joint pain, while bone signal changes were detectable in the cases with advanced OA with joint pain [6]. In this study, signal changes were seen even in the cases without $\mathrm{OA}$ or with a slight degree of $\mathrm{OA}$ if the patients had joint pain. In most cases, the joint pain improved, but the patients progressed to further-stage OA. Therefore, we propose that OA classification can be evaluated not only by radiographic KL grading scale, but 


\section{(1)Healthy joints (not affected joints)}

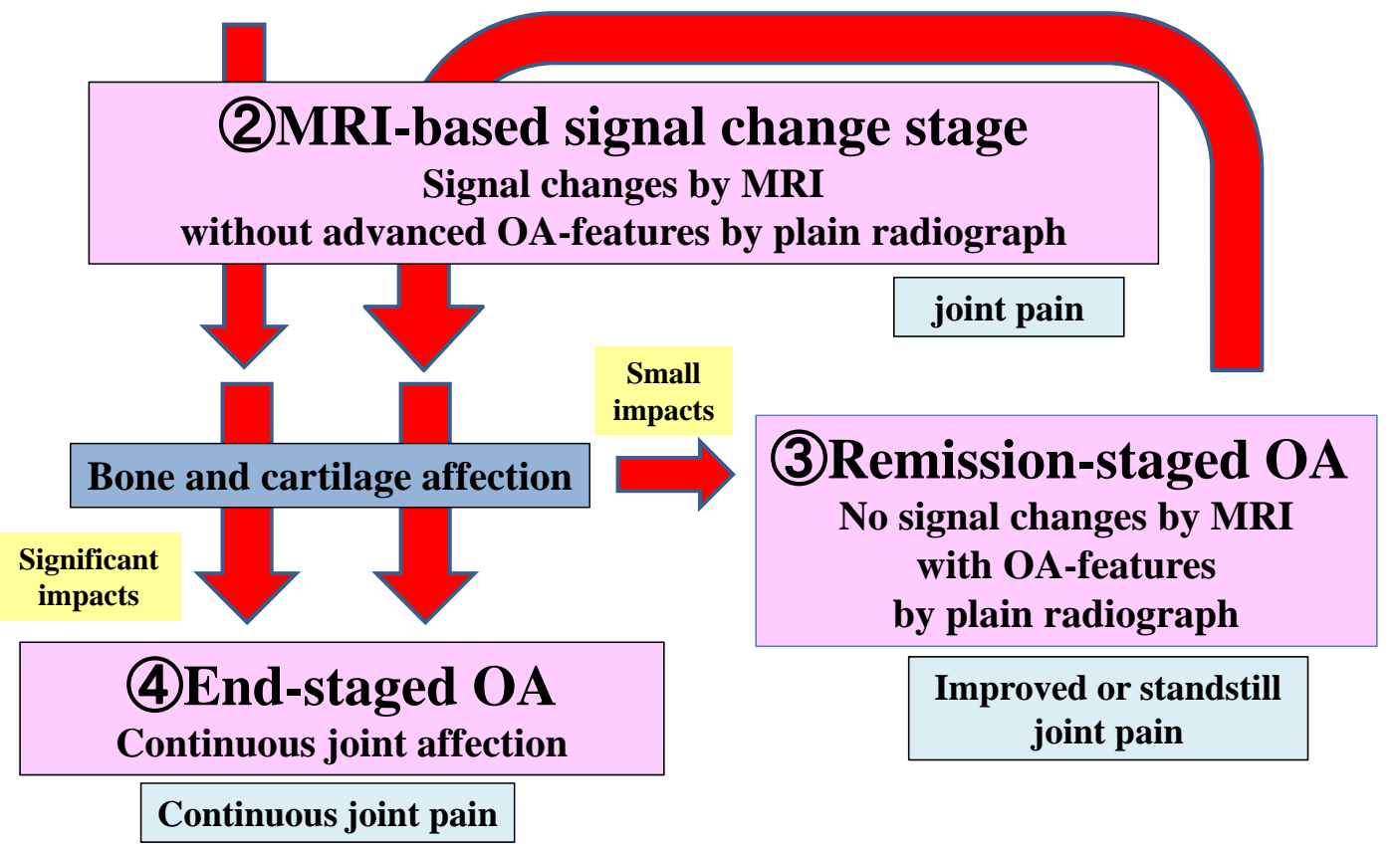

Fig. (7). A possible mechanism of OA progression, which we propose.

also by a new methodology including MRI findings and joint pain.

Hayami et al. describe that Alendronate, a representative $\mathrm{BP}$, inhibits vascular invasion into calcified articular cartilage, leading to the reduction of cartilage degeneration. Alendronate also gives an effect on subchondral bone remodeling, which is the key role on pathogenesis for OA [15]. Iwamoto et al., also review that risedronate, another $\mathrm{BP}$, is effective for knee OA. The authors report that risedronate preserves the structural integrity of subchondral bone [16]. As we describe in this study, microfractures seem to contribute to the pathogenesis of OA. Therefore, we speculate that BP can improve or prevent OA by means of prevention of bone microfractures. Bellido et al. have previously reported that coexist of osteoporosis and osteoarthritis aggravates subchondral bone microstructural damage [17]. Taken together, our study and previously reported studies suggest that osteoporosis treatment could help to prevent OA progression.

The current study has a limitation in that a small number of cases was examined ( 7 cases). Further studies with larger numbers of cases, where the correlation between MRI findings and hip joint pain are evaluated, are therefore necessary. Therefore, we cannot conclude that all cases would proceed to further-stage OA. Further observation will be needed for those cases to confirm this study.

\section{CONFLICT OF INTEREST}

The authors confirm that this article content has no conflicts of interest.

\section{ACKNOWLEDGEMENTS}

Declared none.

\section{REFERENCES}

[1] Radin EL, Rose RM. Role of subchondral bone in the initiation and progression of cartilage damage. Clin Orthop Relat Res 1986; 213: 34-40.

[2] Burr DB, Gallant MA. Bone remodelling in osteoarthritis. Nat Rev Rheumatol 2012; 8: 665-73.

[3] Guermazi A, Roemer FW, Haugen IK, Crema MD, Hayashi D. MRI-based semiquantitative scoring of joint pathology in osteoarthritis. Nat Rev Rheumatol 2013; 9: 236-51.

[4] Xu L, Hayashi D, Roemer FW, Felson DT, Guermazi A. Magnetic resonance imaging of subchondral bone marrow lesions in association with osteoarthritis. Semin Arthritis Rheum 2012; 42: $105-18$.

[5] Guermazi A, Niu J, Hayashi D, et al. Prevalence of abnormalities in knees detected by MRI in adults without knee osteoarthritis: population based observational study (Framingham Osteoarthritis Study). BMJ 2012; 345: e5339.

[6] Kamimura M, Nakamura Y, Ikegami S, Uchiyama S, Kato H. Joint pain undergoes a transition in accordance with signal changes of bones detected by MRI in hip osteoarthritis. Open Rheumatol J 2013; 7: 58-65.

[7] Muraki S, Oka H, Akune T, et al. Prevalence of radiographic knee osteoarthritis and its association with knee pain in the elderly of Japanese population-based cohorts: the ROAD study. Osteoarth Cartil 2009; 17: 1137-43.

[8] Denis F, Armstrong GW, Searls K, Matta L. Acute thoracolumbar burst fractures in the absence of neurologic deficit. A comparison between operative and nonoperative treatment. Clin Orthop Relat Res 1984; 189: 142-9.

[9] Takahara K, Kamimura M, Nakagawa H, Hashidate H, Uchiyama S. Radiographic evaluation of vertebral fractures in osteoporotic patients. J Clin Neurosci 2007; 14: 122-6. 
[10] Stafford SA, Rosenthal DI, Gebhardt MC, Brady TJ, Scott JA. MRI in stress fracture. AJR Am J Roentgenol 1986; 147: 553-6.

[11] Yao L, Lee JK. Occult intraosseous fracture: detection with MR imaging. Radiology 1988; 167: 749-51.

[12] Burr DB, Schaffler MB. The involvement of subchondral mineralized tissues in osteoarthrosis: quantitative microscopic evidence. Microsc Res Tech 1997; 37: 343-57.

[13] Burr DB. The importance of subchondral bone in osteoarthrosis. Curr Opin Rheumatol 1998; 10: 256-62.

[14] Burr DB, Radin EL. Microfractures and microcracks in subchondral bone: are they relevant to osteoarthrosis? Rheum Dis Clin North Am 2003; 29: 675-85.
[15]

Hayami T, Pickarski M, Wesolowski GA, et al. The role of subchondral bone remodeling in osteoarthritis: reduction of cartilage degeneration and prevention of osteophyte formation by alendronate in the rat anterior cruciate ligament transection model. Arthritis Rheum. 2004;50:1193-206.

[16] Iwamoto J, Takeda T, Sato Y, Matsumoto H. Effects of risedronate on osteoarthritis of the knee. Yonsei Med J. 2010;51:164-70.

[17] Bellido M, Lugo L, Roman-Blas JA, et al. Subchondral bone microstructural damage by increased remodelling aggravates experimental osteoarthritis preceded by osteoporosis. Arthritis Res Ther 2010; 12(4): R152.

(c) Kamimura et al.; Licensee Bentham Open.

This is an open access article licensed under the terms of the Creative Commons Attribution Non-Commercial License (http://creativecommons.org/licenses/by-nc/ $3.0 /$ ) which permits unrestricted, non-commercial use, distribution and reproduction in any medium, provided the work is properly cited. 